\title{
Composição química do óleo essencial do fruto da Mangabeira (Hancornia speciosa
}

\section{Gomes) do Tocantins}

\author{
Chemical composition of the essential oil of the Mangabeira (Hancornia speciosa Gomes) fruit from \\ Tocantins
}

Composición química del aceite esencial del fruto de Mangabeira (Hancornia speciosa Gomes) de

Tocantins

Recebido: 08/02/2022 | Revisado: 15/02/2022 | Aceito: 19/02/2022 | Publicado: 01/03/2022

\author{
Jacqueline Soares Carvalho \\ ORCID: https://orcid.org/0000-0001-9920-3024 \\ Universidade Federal do Tocantins, Brasil \\ E-mail: jacqueline_carvalho@uft.edu.br \\ Flamys Lena do Nascimento Silva \\ ORCID: https://orcid.org/0000-0002-8284-8608 \\ Universidade Federal do Norte do Tocantins, Brasil \\ E-mail: flsilva@uft.edu.br \\ Edenilson dos Santos Nicolau \\ ORCID: https://orcid.org/0000-0003-0326-5795 \\ Universidade Federal do Norte do Tocantins, Brasil \\ E-mail: enicolau@uft.edu.br \\ Michele Cristiane Diel Rambo \\ ORCID: https://orcid.org/0000-0002-5329-4933 \\ Instituto Federal de Educação, Ciência e \\ Tecnologia do Tocantins, Brasil \\ E-mail: michele.rambo@ifto.edu.br \\ Leandro Ferreira da Silva \\ ORCID: https://orcid.org/0000-0002-6163-4002 \\ Universidade Federal do Tocantins, Brasil \\ E-mail: leandroferreira@uft.edu.br \\ Magale Karine Diel Rambo \\ ORCID: https://orcid.org/0000-0003-2529-9574 \\ Universidade Federal do Tocantins, Brasil \\ E-mail: magalerambo@uft.edu.br
}

\begin{abstract}
Resumo
A mangaba é um fruto da mangabeira (Hancornia speciosa Gomes), uma árvore nativa do cerrado brasileiro, com grande potencial alimentício e medicinal. Neste trabalho foi realizado o estudo da composição química do óleo essencial do fruto maduro mangaba pertencentes as regiões de Lajeado e Filadélfia do estado do Tocantins. Os experimentos foram realizados no Laboratório de Cromatografia (LABCROM-UFT), Campus de Araguaína, unidade Cimba. Para obtenção dos perfis químicos, foram realizados dois métodos de extração (hidrodestilação e partição líquido - líquido) com separação e identificação dos compostos dos voláteis por cromatografia a gás acoplada a espectrometria de massas (GC-MS). Os resultados do perfil químico volátil do óleo essencial da mangaba apontam a presença de funções ésteres (salicilato de metila, benzoato de etila), álcoois (linalol, álcool feniletílico, citronelol, geraniol, eugenol, álcool benzílico), ácidos carboxílicos (ácido fenil propanoico, ácido 2,4-dimetil-pentanoico), hidrocarboneto ( decano, 2,5 ciclohexadieno), aldeídos (undecanal, dodecanal, acelaldeído benzeno), cetonas (acetofenona), sendo os álcoois a classe de compostos mais abundantes no óleo essencial. A pesquisa mostrou o potencial da fruta na área da indústria alimentícia, farmacêutica e cosmética, além da exploração sustentável e preservação biológica da mangabeira e seu fruto.
\end{abstract}

Palavras-chave: Cerrado; Mangaba; Perfil químico; Voláteis.

\begin{abstract}
Mangaba is a fruit of the mangabeira (Hancornia speciosa Gomes), a originated from the Brazilian cerrado, with alimentary and medicinal potential. The aim of this work was to analyses the chemical composition of aroma essential oil of mangaba fruit from the regions of Lajeado and Filadélfia of the state Tocantins. Analyses were performed at the Chromatography Laboratory (LABCROM-UFT) of the Federal University of Tocantins Campus Araguaína-Cimba. To get the chemical profiles two methods of extraction were carried out (hydrodestillation and liquid-liquid), separation and identification of the volatile compounds of essential oil were by gas chromatography coupled to mass
\end{abstract}


spectrometry (GC-MS). The results of the chemical profile of mangaba essential oil showed the presence of esters (methyl salicylate, ethyl benzoate), alcohols (linalool, phenylethyl alcohol, citronellol, geraniol, eugenol, benzyl alcohol), carboxylic acids (benzene propanoic acid, 2,4-dimethyl-pentanoic acid), hydrocarbons (decane, 2,5 cyclohexadiene), aldehydes (undecanal, dodecanal, benzene acelaldehyde), ketones (acetophenone). Alcohols being the abundant specific compounds in essential oil. The research showed the potential of this fruit in the food, pharmaceutical and cosmetic industry, as well as the sustainable explotation and biological preservation of the mangabeira and its fruit.

Keywords: Cerrado; Mangaba; Chemical profile; Volatile.

\section{Resumen}

La mangaba es un fruto de la mangabeira (Hancornia speciosa Gomes), un árbol originario del Cerrado brasileño, con gran potencial nutricional y medicinal. En este trabajo se realizó el estudio de la composición química del aceite esencial del fruto maduro de mangaba perteneciente a las regiones de Lajeado y Filadélfia del estado de Tocantins. Los experimentos se realizaron en el Laboratorio de Cromatografía (LABCROM-UFT), Campus Araguaína, unidad Cimba. Para la obtención de los perfiles químicos se realizaron dos métodos de extracción (hidrodestilación y partición líquido-líquido) con separación e identificación de compuestos volátiles por cromatografía de gases acoplada a espectrometría de masas (GC-MS). Los resultados del perfil químico volátil del aceite esencial de mangaba indican la presencia de funciones éster (salicilato de metilo, benzoato de etilo), alcoholes (linalool, alcohol feniletílico, citronelol, geraniol, eugenol, alcohol bencílico), ácidos carboxílicos (ácido fenilpropanoico, 2, ácido 4-dimetilpentanoico), hidrocarburo (decano, 2,5-ciclohexadieno), aldehídos (undecanal, dodecanal, benceno acelaldehído), cetonas (acetofenona), siendo los alcoholes la clase de compuestos más abundante en el aceite esencial. La investigación mostró el potencial de la fruta en el área de la industria alimenticia, farmacéutica y cosmética, además de la explotación sustentable y la preservación biológica de la mangabeira y su fruta.

Palabras clave: Cerrado; Mangaba; Perfil químico; Volátil.

\section{Introdução}

Mangaba (Hancornia speciosa GOMES, Apocynaceae) é uma fruta da região tropical brasileira com aroma e sabor exóticos. Os frutos são do tipo baga, de tamanho, forma e cores variados, geralmente elipsoidais ou arredondados, variando de 2,5 a 6,0 cm de diâmetro, exocarpo amarelado ou esverdeado com pigmentação vermelha ou sem pigmentação, polpa amarela, bastante suave, adocicada, carnoso- viscosa e ácida, contendo de 2 a 30 unidades de sementes discoides, achatadas e com coloração castanho-claro, de 7 a 8 mm de diâmetro (Ganga et al. 2009).

A mangaba apresenta boa digestão e valor nutritivo, com teor de proteína superior ao da maioria das frutas (Ganga et al. 2009; Venturini Filho 2010). Existe um enorme potencial para exploração desta fruta nativa no setor agroindustrial brasileiro, na fabricação de polpa, suco, sorvete, licor e doces (Silva Junior 2004; Ferreira 2009). A casca, folha e raiz da árvore mangabeira têm aplicação na medicina popular, no tratamento de úlceras e gripes (Almeida et al. 1998; Alves 2014).

Os compostos voláteis são os responsáveis pelo aroma e sabor dos alimentos. Contudo, a composição química, incluindo a distribuição dos compostos do aroma depende da espécie, das condições ambientais, estágios de maturação e região de cultivo (Simões et al. 1999; Jakiemiu 2008). A mangaba é rica em um óleo essencial de aroma característico composto de várias classes químicas distintas tais como ésteres, álcoois, ácidos carboxílico, fenóis, cetonas etc. Além disso, estas substâncias conferem ao fruto características específicas como cor, sabor e outras propriedades que variam consideravelmente de espécie para espécie, em função de parâmetros climáticos e de fatores agronômicos, como fertilização, irrigação e, especialmente, a fase de desenvolvimento na planta durante a colheita (Paviani, 2004).

O desenvolvimento desse trabalho com o óleo essencial da mangaba vem ao encontro de conhecê-la e principalmente divulgar o potencial desse recurso natural que é o fruto do cerrado tocantinense, visando à exploração sustentável e a preservação biológica da planta e fruto. Foram realizados os estudos da composição química do óleo essencial da mangaba madura pertencentes aos municípios de Lajeado e Filadélfia no Tocantins. Os perfis químicos foram obtidos por dois métodos de extração dos compostos voláteis com o processo de hidrodestilação, em seguida, foi realizado as partições líquido-líquido e a separação e identificação compostos voláteis por cromatografia a gás acoplada a espectrometria de massas (GC-MS). 


\section{Metodologia}

\subsection{Local do Experimento}

A parte experimental foi realizada no Laboratório de Cromatografia do curso de licenciatura em química da Universidade Federal do Tocantins (LABCROM-UFT), Campus-Cimba em Araguaína.

\subsection{Amostras}

Foram coletados três quilogramas $(3 \mathrm{Kg})$ de mangabas maduras em dois municípios do estado do Tocantins em períodos diferentes. A primeira coleta ocorreu nos pomares do município de Lajeado no mês de novembro de 2017, enquanto que a segunda coleta ocorreu no município de Filadélfia em outubro de 2018. Após a coleta, os frutos foram devidamente higienizados e congelados.

\subsection{Extrações dos compostos voláteis}

A extração dos compostos voláteis foi realizada empregando a técnica de hidrodestilação, iniciado após o processo de maceração dos frutos maduros.

Na hidrodestilação utilizou-se um aparelho denominado Clevenger. Neste sistema empregou-se um balão de fundo redondo $(1000 \mathrm{~mL})$ contendo cerca de $300 \mathrm{~g}$ de polpa de mangaba, que foi inicialmente preenchido com água destilada $(600 \mathrm{~mL})$. O balão foi acoplado ao sistema de destilação e depois aquecido até a temperatura de ebulição, permanecendo assim por 3 horas.

O extrator foi montado para funcionar em circuito fechado, desta forma as perdas de substâncias voláteis foram minimizadas. Assim os compostos voláteis foram arrastados pelo vapor e condensados juntamente com a água.

\subsection{Partições líquido - líquido}

Após a destilação, iniciou-se o processo de partição líquido - líquido para separar o óleo essencial do produto condensado. Para a partição líquido - líquido foram misturados em funil de separação volumes iguais de $140 \mathrm{~mL}$ de acetato de etila (grau HPLC, 99,9 \% de pureza) e hidrolato (1:1). Em seguida, agitou-se a mistura para a liberação do gás e deixou-se em repouso durante cerca de cinco minutos. Depois disso, foi possível observar a separação entre o hidrolato (120 mL) e a fase orgânica $(180 \mathrm{~mL})$ contendo o óleo essencial. Posteriormente, reservou-se a fase orgânica e repetiu-se o processo de mistura do hidrolato obtido da primeira separação com Acetato de Etila (120 mL de cada).

O procedimento realizado foi o mesmo para as duas diferentes amostras de mangaba do estado do Tocantins. Contudo, o volume inicial da amostra do município de Lajeado foi de $130 \mathrm{~mL}$.

Seguindo a separação, filtrou-se a fase orgânica utilizando $\mathrm{Na}_{2} \mathrm{SO}_{4}$ sal anidrido para secagem e colocou se no roto evaporador onde o solvente e o óleo essencial foram separado.

Finalizado o processo de rota evaporação, a massa final de mangaba obtida do município de Filadélfia foi de $0,1173 \mathrm{~g}$ (rendimento de $0,04 \%$ ). Ao passo que a massa final de mangaba obtida do município de Lajeado foi igual a $0,1272 \mathrm{~g}$ (rendimento de $0,04 \%$ ) após secagem na capela de exaustão química. 
Figura 1. Fluxograma da parte experimental.

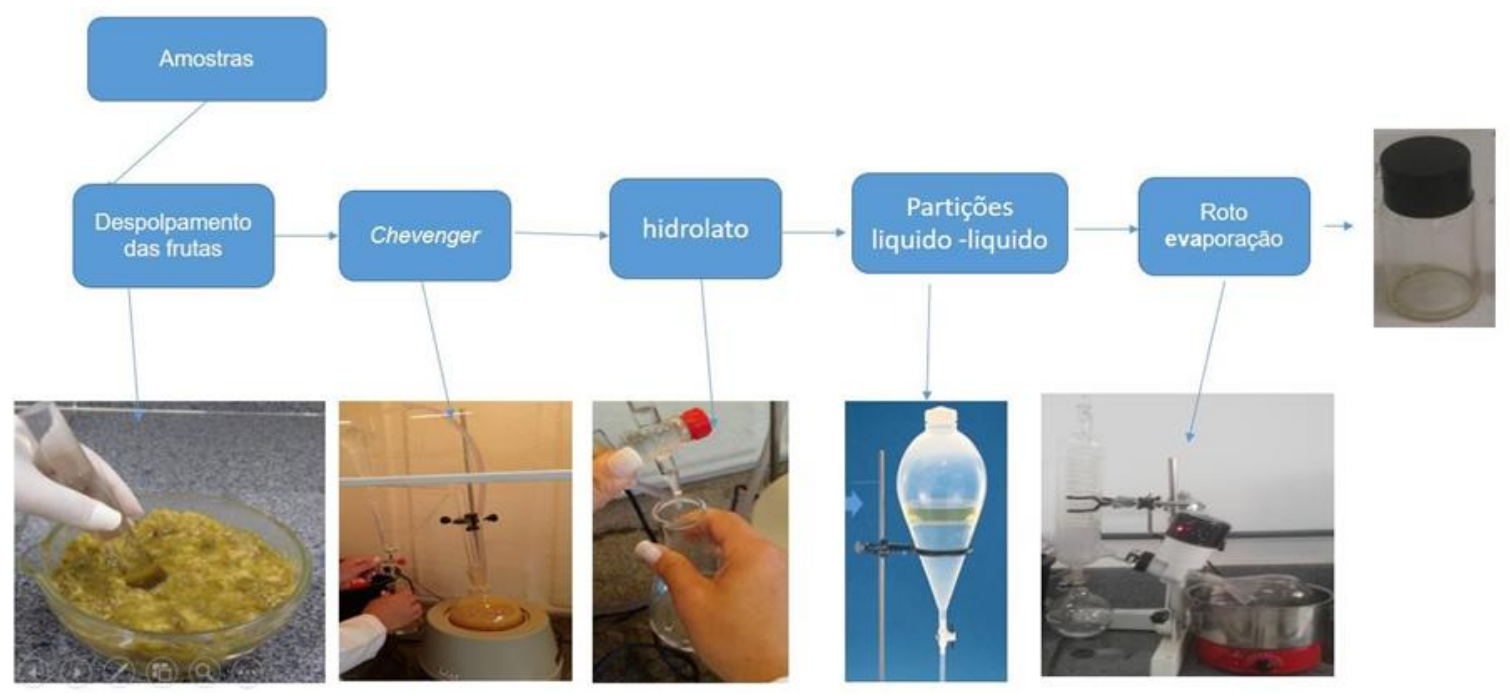

Fonte: Elaborado pelos autores (2022).

\subsection{Análises dos compostos voláteis do óleo essencial de Hancornia speciosa por GC-MS.}

No preparo da amostra, pesou-se cerca de 0,0004 g do óleo essencial da mangaba do município de Lajeado, diluiu-se em $4 \mathrm{~mL}$ de Acetato de Etila. Em seguida, utilizou-se um filtro de membrana PTFE $0.20 \mu \mathrm{m}$ e seringa de $5 \mathrm{~mL}$ para filtrar a amostra. O filtrado foi transferido diretamente para um vial de vidro de $1,5 \mathrm{~mL}$, que foi colocado no mostrador automático do cromatógrafo a gás Agiltent Techologies (modelo Shimadzu QP7890B) com espectrômetro de massas (modelo Shimadzu 5977 B) equipado com uma coluna capilar de sílica fundida J \& W Scientific HP-5MS, contendo 5\% de fenil metil siloxano (30 m de comprimento $\times 0,25 \mathrm{~mm} \times 0,25 \mu \mathrm{m}$ de espessura do filme) a programação do forno foi: temperatura inicial $50{ }^{\circ} \mathrm{C}$ (por 1 min), $50{ }^{\circ} \mathrm{C}$ a $230{ }^{\circ} \mathrm{C}\left(10{ }^{\circ} \mathrm{C} / \mathrm{min}\right), 230{ }^{\circ} \mathrm{C}$ a $280{ }^{\circ} \mathrm{C}\left(25^{\circ} \mathrm{C} / \mathrm{min}\right)$ permanecendo em $280{ }^{\circ} \mathrm{C}$ por 10 minutos. O gás Hélio foi usado como gás de arraste, com o fluxo constante $1,2 \mathrm{~mL} / \mathrm{min}$.

$\mathrm{O}$ volume injetado da amostra foi $1,0 \mu \mathrm{L}$, modo splitless. O detector de massas operou nas as seguintes condições: energia de ionização: $70 \mathrm{eV}$, e as temperaturas da interface: $280^{\circ} \mathrm{C}$, temperatura do quadrupolo: $150{ }^{\circ} \mathrm{C}$, sendo o tempo da corrida de 31 minutos. Na mesma programação de temperatura, a mistura padrão de n-alcano (C10- C30) foi analisado.

\subsection{Processos de Identificação dos compostos do óleo essencial Hancornia speciosa}

O índice de Kovart's foi calculado e comparado com índice de retenção da literatura (Fluxograma 2). O índice de retenção é baseado na equação de Van den Dool \& Kratz (1963), pela Equação 1:

$$
I R=100 i x \frac{(T R R-T R H A)}{\text { TRHD } T R H A}+100 \mathrm{~N} \text { Equação 1' ‘ }
$$

Onde:

TRX = tempo de retenção do composto $(X)$;

TRHA = tempo de retenção do hidrocarboneto que elui antes do composto (HA);

TRHD = tempo de retenção do hidrocarboneto que elui depois do composto (HD);

$i=$ diferença entre o número de carbono que elui antes (HA) e depois (HD);

$N=$ número de carbonos do hidrocarboneto que elui antes (HA). 
Os compostos foram identificados usando a literatura (Adams, 2007) o Programa de Pesquisa Espectral de Massas da biblioteca NIST 2014 (Instituto Nacional de Padrões de Tecnologia, Washington, DC, EUA) e para calcular o índice de retenção, utilizou como base os intervalos do n-alcano C10-C30.

\section{Resultados e Discussões}

Através das análises da composição química do óleo essencial da mangaba foi possível identificar a presença das funções químicas ésteres, álcoois, ácidos carboxílicos, éteres, acetona e aldeídos.

$\mathrm{Na}$ amostra do município de Lajeado foram identificados 33 compostos voláteis, dos quais 10 são majoritários. Enquanto na amostra do município de Filadélfia foram encontrados 33 compostos, sendo 11 majoritários. O cromatograma obtido da amostra de Lajeado é mostrado na Figura 2. Nele é possível observar a presença de diversos sinais analíticos (picos), que correspondem aos compostos voláteis identificados. Em destaque do lado direito do cromatograma estão listados os compostos majoritários segundo sua ordem de eluição, destacando-se em percentual de área acetofenona 4,65\%.

Figura 2. Cromatograma do óleo essencial de mangaba do município de Lajeado.

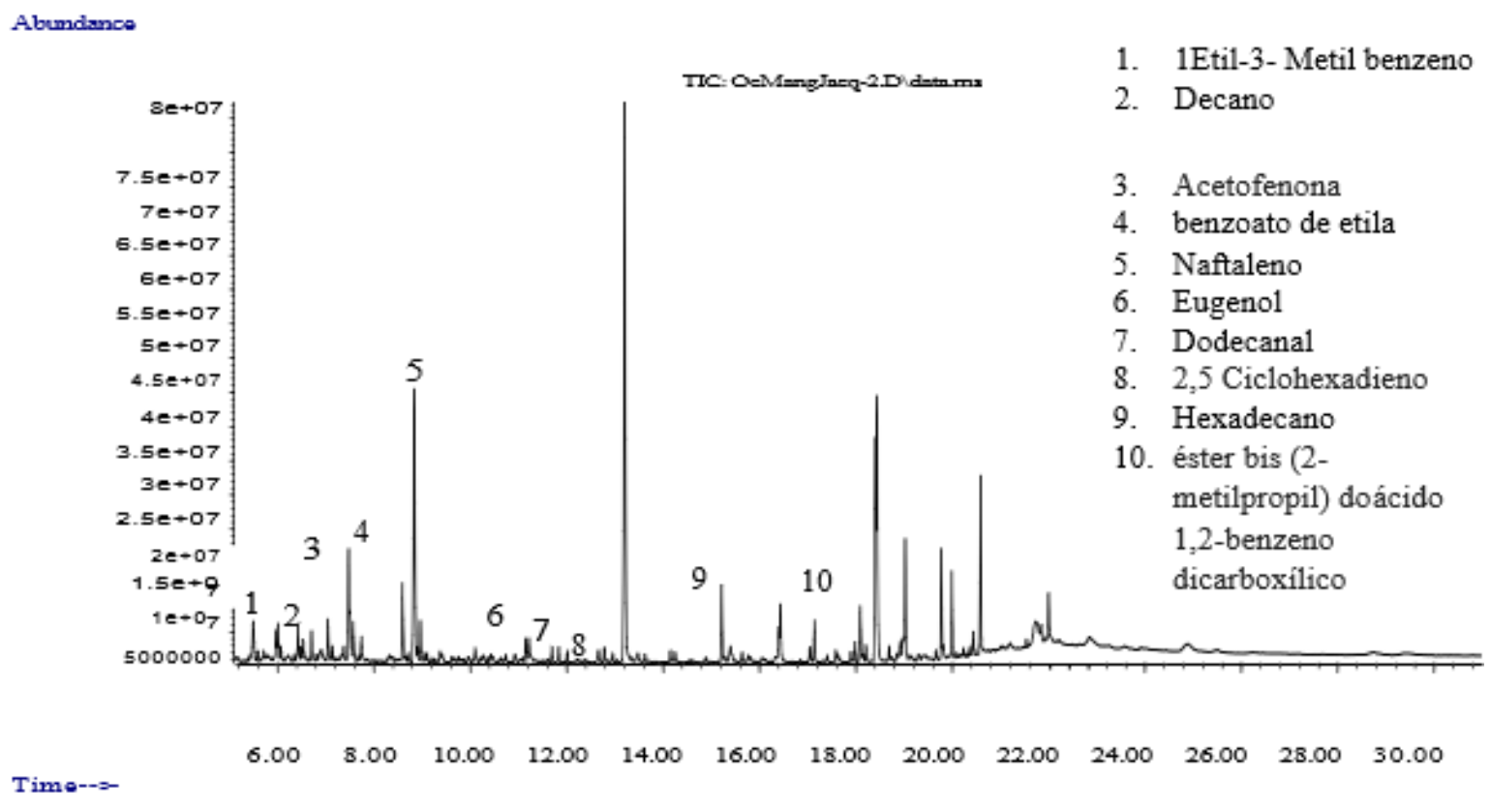

Fonte: Elaborado pelos autores (2022).

Sampaio (2008) ao analisar componentes voláteis dos frutos maduros, evidenciou diferenças significativas, onde identificou 34 compostos, sendo 11 majoritários, dos quais dois não são observados nesse estudo como: pentanal e furfural. Dos compostos identificados por Sampaio (2008) apenas 09 e assemelham com o encontrado nesta pesquisa, que pode ser justificado por se tratar do mesmo fruto, as amostras são diferentes; colhidas em regiões distintas, extraídas com solvente diferente e a programação de temperatura utilizada no cromatógrafo não é a mesma, esses fatores influência nos resultados.

No cromatograma do óleo essencial de mangaba do município de Filadélfia (Figura 3) observa-se uma redução no número de compostos identificados. Dentre os compostos majoritários identificados estão linalol $2,98 \%$, álcool feniletílico $1,97 \%$, citronelol $1,03 \%$, geraniol 1,06 \%, eugenol 3,96\%, álcool benzílico 0,93\%. 
Figura 3. Cromatograma do óleo essencial de mangaba do município de Filadélfia.

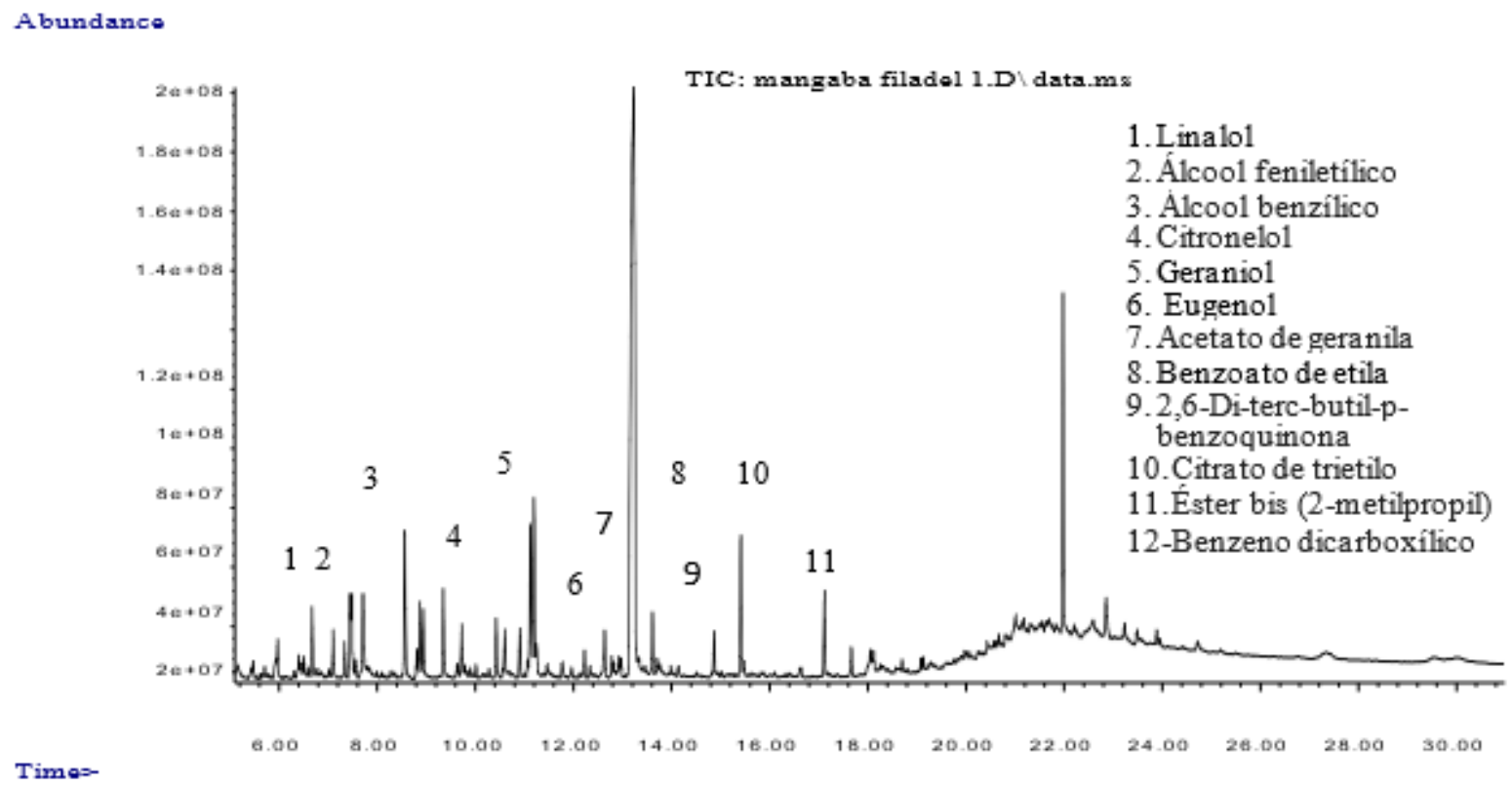

Fonte: Elaborado pelos autores (2022).

De acordo com os dados obtidos, o óleo essencial apresentou sinais de contaminação, tanto por solvente quanto na amostra, como pode ser observado no cromatograma da figura 4. A análise do solvente (branco Anexo C) foi realizada nas mesmas condições que o óleo essencial da mangaba, e neste observou- se a presença dos contaminantes: Acrilato de Dodecilo e Fitalato de di-isooctila, já o contaminante da amostra foi fosfato de tributila. Estes são plastificantes que aparecem como sinais bem intensos nos cromatogramas. $\mathrm{O}$ fato de ter encontrado contaminação nas amostras não prejudicou os resultados do trabalho, pois os tempos de retenção destes eventuais contaminantes são diferentes dos compostos voláteis do óleo essencial, com isso também permitiu-se comprovar a eficiência e confiabilidade da técnica analítica de identificação.

Figura 4. Cromatograma da amostra do óleo essencial de mangaba do município de Lajeado com contaminação.

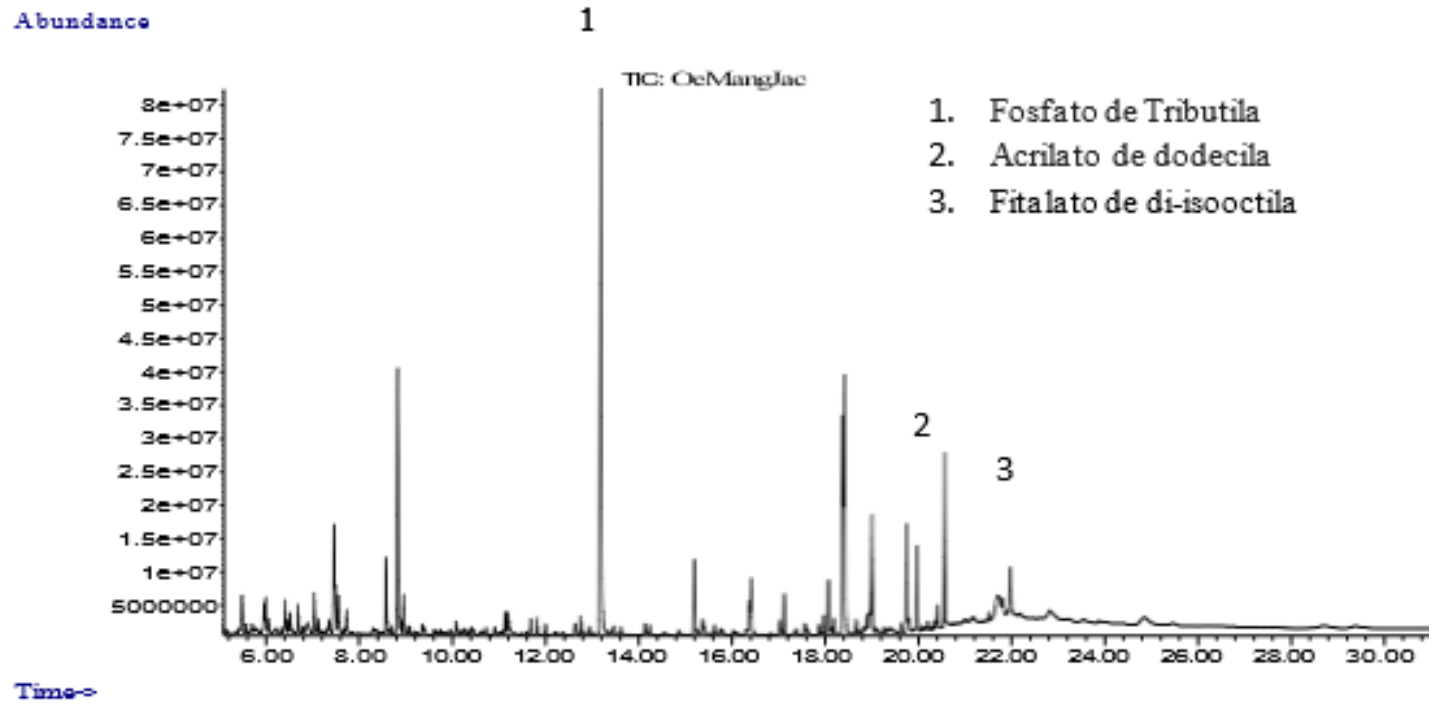

Fonte: Elaborado pelos autores (2022).

\section{Conclusão}

Os onze compostos comuns identificados no óleo essencial da mangaba dos dois municípios foram: 1-etil-3-metil- 
benzeno, 1,2,4-trimetil-benzeno, linalol, álcool feniletílico, éster metílico do ácido benzóico, $\alpha$-Terpineol, salicilato de metila, 1- fenoxipropan-2-ol, geraniol, dibutilformamida e eugenol. A principal classe química observada foi álcool.

O óleo essencial extraído das duas amostras de mangaba apresentou pouca semelhança, esse fato pode ser comprovado quando analisamos a quantidade de compostos comuns encontrados, essa diferença pode ter sido ocasionada pelo tipo de solo e diferença de localidade das plantas, como também pelo tempo de evaporação ocorrido na capela de exaustão química após a extração.

Foram encontrados nas análises os compostos linalol, geraniol, eugenol, estes estão geralmente presentes na maioria dos óleos essenciais extraídos de flores, frutos, raízes e sementes. Este fato comprova a qualidade do atual método de extração e caracterização do óleo essencial da mangaba com os da literatura, devido à similaridade entre a constituição química volátil.

Nas pesquisas realizadas anteriormente com as outras partes da mangabeira como cascas e folhas, foram encontrados compostos químicos com características analgésica e anti-inflamatória (Nogueira, et al.2004), e essas mesmas são encontradas no óleo essencial do fruto, tais como eugenol, salicilato de metila.

Para o futuro, sugerimos estudos e análises de triacilgliceróis (TAGs) do óleo de mangaba. A grande importância na determinação dessas moléculas se deve ao fato de serem as principais constituintes da composição de óleos e gorduras de origem natural e vegetal, e possuem alto potencial de uso como produtos alimentício, farmacêutica e comercial. Essas moléculas podem ser determinadas utilizando as técnicas cromatográficas, tais como a Cromatografia líquida de alta eficiência acoplada a Espectrometria de Massas (HPLC-MS) e Cromatografia gasosa com detector de chama (GC-FID) (Yang et al. 2013; Lall et al. 2009; Jabeur et al. 2014).

\section{Considerações Finais}

Com a aplicação da técnica de GC-MS foi possível obter o perfil químico volátil do óleo essencial da mangaba pertencente as regiões de Lajeado e Filadélfia -To. Esta ferramenta analítica permitiu identificar a presença de ésteres, álcoois, ácidos carboxílicos, aldeídos, cetona, dos quais os álcoois foram as substâncias químicas mais presentes no óleo essencial. Os compostos com maior percentagem de área, comuns as duas amostras, que podem ser os responsáveis pelo aroma característico do óleo essencial da fruta são: linalol, álcool feniletílico, benzoato de etila, citronelol, geraniol, acetato de geranila, álcool feniletílico, 2,6-di-terc-butil-p-benzoquinona e citrato de trietilo.

A pesquisa desenvolvida teve um caráter investigativo do perfil químico do óleo essencial da maganba, para um maior conhecimento do potencial dessa fruta na área alimentícia, farmacêutica e de cosmético, visando à exploração sustentável e a preservação biológica mangabeira e seu fruto.

O autor que fornece suporte metodológico é o Professor Doutor Marcos Nogueira Eberlin, através dos trabalhos "Brazil nut oil: quality control via triacylglycerol profiles provided by easy ambient sonic-spray ionization mass spectrometry" e "Amazonian vegetable oils and fats: fast typification and quality control via triacylglycerol (TAG) profiles from dry matrixassisted laser desorption/ionization time-of-flight (MALDI-TOF) Mass Spectrometry Fingerprinting" (Funasaki et al. 2012; Saraiva et al. 2009).

\section{Referências}

Adams, R. P. (2012). Identification of essential oil components by gas chromatography/mass spectrometry. Allured Business Media.

Affonso, R. S., Rennó, M. N., Slana, G. B. C. A. \& França, T. C. C. (2012). Aspectos Químicos e Biológicos do Óleo Essencial de Cravo da Índia. Revista Virtual Química, 4. 146-161.;

Andria \& Maharavo (2014). N.R. Retention Data. NIST Mass Spectrometria Data Center. NIST massSpectrometry Data Center. 
Almeida, C. C. S., Espíndola, A. C. M., Carvalho, N. S. G. \& Silva, M. S. (2003). Variabilidade genética em mangabeira estimada através de caracteres morfológicos. In: Simpósio Brasileiro Sobre A Cultura Da Mangaba, Aracaju - SE, 2003. Aracaju: Embrapa tabuleiros Costeiros, CD-ROM.

Aloufa, M. A. (2003). Multiplicação e conservação in vitro de mangabeira. In: Simpósio Brasileiro Sobre A Cultura Damangaba, Aracaju-SE, 2003. Aracaju: Embrapa Tabuleiros Costeiros. CD-ROM.

Asuming, W. A., Beauchamp, O. S., Descallzo, J. T., Dev, V. F. S. \& Ma, C. W. (2005). Composition of the essential oil of four Lomatium Raf. Specie sand their chemotaxonomy. Biochemical System Ecology, 331, 17-26.

Carvalho, M. G., Velloso, C. R. X., Braz-Filho, R. \& Costa, W. F. (2001) Acyl-lupeol Ester from Parahancorniaa mapa (Apocynaceae). Journal of the Brazilian Chemical Society, 12, 556-559.

Carvalho, G. A. (2015). Determinação de resíduos de agrotóxicos em mamão empregando o método Qhehers e GM-MS, 2015, 127f. Dissertação em Química, Universidade Federal da Bahia - UFBA.

Cassel, E. \& Vargas, R. M. F. (2006). Experiment with sand modeling of the common paper graffiti in the extraction of essential oil by steam distillation. Journal Mexican Chemistry Society, 55, 57-60.

Cassel, E ., Vargas, R. M. F. \& Brun, G. W. (2008). Processos de extração supercrítica aplicados a produtos naturais. In: Fundamentos de Tecnologia de Produtos Fitoterapéuticos. Porto Alegre: EDIPUCRS.

Collis C. H, Braga, G. L. \& Bonatos, P.S. (2006). Fundamento de Cromatografia. Unicamp.

Costa, M. A. P. C. et al. (2003). Principais resultados com micro propagação de mangabeira na Bahia. In: Simpósio Brasileiro Sobre A Cultura Da Mangaba, Aracaju - SE. Embrapa Tabuleiros Costeiros. CD- ROM.

Cronquist, A. (1988). The evolution and classification of flowering plants. (2a ed.), The New York Botanical Garden.

Danisco, P. P. (2001). Comparing extraction by traditional solvents with supercritical extraction from in economical environmental stand point. ISASF - CDROM. Versailles, France.

Durman, G., Okutucu, C., Ucar, S., Stahl, R. \& Yanik, J. (2011). The slow and fast pyrolysis of cherry seed. Bioressource Technology, $102,1869-1878$.

Ferhat, M. A., Tigrine-Kordjani, N., Chemat, S., Meklati, B. Y., \& Chemat, F. (2007). Rapid Extraction of Volatile Compounds Using a New Simultaneous Microwave Distillation: Solvent Extraction Device. Chromatographia, 65(3-4), 217-222.

Ferreira, M. B. (1980). Frutos comestíveis nativos do cerrado. Informe Agropecuário,.6, 13-21.

Ferreira, E. G. (2009). Produção integrada de mangaba. In: Produção integrada no Brasil agropecuária sustentável alimentos seguros. Ministério Da Agricultura, Pecuára E Abastecimento. Secretaria De Desenvolvimento Agropecuário E Cooperativismo., capitulo 22.

Ferreira, E. G. \& Marinho, S. O. (2007). Produção de frutas da mangaba para o consumo in natura e industrializado. Tecnologia e Ciências. Agropecuárias v. $1,9-14$.

Fonseca, A. A. O. et al. (2003). Otimização da formulação de néctar de mangaba. In: Simpósio Brasileiro Sobre A Cultura Da Mangaba, Aracaju - Se. Embrapa Tabuleiros Costeiros. CD-ROM.

Funasaki, M., Oliveira, R. S., Zanotto, S. P., Carioca, C. R. F., Simas, R. C., Eberlin, M. N., \& Alberici, R. M. (2012). Brazil Nut Oil: Quality Control via Triacylglycerol Profiles Provided by Easy Ambient Sonic-Spray Ionization Mass Spectrometry. Journal of Agricultural and Food Chemistry, 60(45), 1126311267.

Ganga, R, M, D., Chaves, L, J. \& Naves, R. V. (2009). Parâmetros genéticos em progênies de Hancornia speciosa Gomes do cerrado. Scientia Forestalis, 37, 395-404.

Guenther, E. (1976) The essential oils. Krieger Publishing Company, 1- 4, 507.

Hui, Y. H. (2007). Handbook of Food Products Manufacturing. Hoboken, Nj, Usa John Wiley \& Sons, Inc.

Ibraf. (2018). Instituto Brasileiro de Fruticultura. Produção Brasileira de Frutas. http://ibraf.org.br/estatisticas/est_frutas.ssp.

Jakiemiu, E. A. R. (2008). Uma contribuição ao estudo do óleo essencial e do extrato de tomilho (Thymus vulgaris L.). 90 f. Dissertação (Mestrado em Tecnologia de Alimentos). Curitiba: Setor de Tecnologia, Universidade Federal do Paraná.

Kader, M.A., Islam, M.R., Parveen, M., Haniu, H. \& Takai, K. (2013). Pyrolysis decomposition of tamarind seed for alternative fuel. Bioresource Technology, v. 149, 1-7.

Kotowska, U., Żalikowski, M., \& Isidorov, V. A. (2011). HS-SPME/GC-MS analysis of volatile and semi-volatile organic compounds emitted from municipal sewage sludge. Environmental Monitoring and Assessment, 184(5), 2893-2907.

Lederman, I. E., Silva Júnior, J. F., Bezerra, J. E. F. \& Espíndola A.C.M. (2000). Mangaba (Hancornia speciosa Gomes). Série Frutas Nativas 2. Sp: FUNEP.

Lima, I. de O., Oliveira, R. de A. G., Lima, E. de O., Farias, N. M. P., \& Souza, E. L. de. (2006). Atividade antifúngica de óleos essenciais sobre espécies de Candida. Revista Brasileira de Farmacognosia, 16(2), 197-201.

Melo, R. C. A. (2005). Plantas Medicinais, óleos essenciais e aromas. Revista Eletrônica Nutritime, 2, 193-200. http://www.nutritime.com.br.

Monachino, J. A. (1945) A revision of Hancornia Speciosa |(Apocynaceae). Lilloa, Tucumán, 11, 19-48. 
Nogueira, P. C. L., Andrade, M. S., Sampaio, T. S., Ribeiro, A. S., Morais, V. R. S., Machado, S. M. F., Alves, P. B., Oliva, G. \& Thiemann, O. H. (2004). Estudo Fitoquímico e avaliação farmacológica das plantas da família Apocynacease e Gutiferae do estado de Sergipe. In Seminário de pesquisa FAP-SE, Aracaju-SE.

Paviani, T. C. (2004). Extração com CO2 a altas pressões e fracionamento do óleo essencial de capim-limão utilizando peneiras moleculares. 2004 . 92 f. Dissertação (Mestrado) - Engenharia de Alimentos, Universidade Regional Integrada do Alto Uruguai e das Missões, Erechim-RS.

Pinheiro, C. S. R., Medeiros, D. N. D., Macêdo, C. E. C. D., \& Alloufa, M. A. I. (2001). Germinação in vitro de Mangabeira (Hancornia speciosa Gomez) em diferentes meios de cultura. Revista Brasileira de Fruticultura, 23(2), 413-416.

Pino, J. A., Mesa, J., Muñoz, Y., Martí, M. P., \& Marbot, R. (2005). Volatile Components from Mango (Mangifera indica L.) Cultivars. Journal of Agricultural and Food Chemistry, 53(6), 2213-2223.

Prestes, O. D., Friggi, C. A., Adaime, M. B., \& Zanella, R. (2009). QuEChERS: um método moderno de preparo de amostra para determinação multirresíduo de pesticidas em alimentos por métodos cromatográficos acoplados à espectrometria de massas. Química Nova, 32(6), 1620-1634.

Rawson, A., Patras, A., Tiwari, B. K., Noci, F., Koutchma, T., \& Brunton, N. (2011). Effect of thermal and non-thermal processing technologies on the bioactive content of exotic fruits and their products: Review of recent advances. Food Research International, 44(7), $1875-1887$.

Roussis, V., Tsoukatou, M., Petrakis, P.V., Chinou, I., Skoula, M., \& Harborne, J. B. (2000). Volatiles constituents of four elichrysum species growing in Greece. Biochemistry System Ecology, v. 28, 163-175.

Sampaio, T. S. \& Nougueira, P. C. L. (2006). Volatile componentes of mangaba fruit (Hancornia speciosa Gomes). At three stages of maturity. Food Chemistry, 606-610.

Sampaio, T. S. (2008). Estudo Fitoquímico de Hancornia speciose Gomes: Isolamento, determinação estrutural e atividade biológica. Dissertação de Mestrado. Universidade Federal de Sergipe, 190.

Santos, R. M. (2014). Production and characterization of Bio-oil from industrial agricultural wastes of mango seed. Dissertação de Mestrado. Universidade Federal de Sergipe, 83.

Saraiva, S. A., Cabral, E. C., Eberlin, M. N., \& Catharino, R. R. (2009). Amazonian Vegetable Oils and Fats: Fast Typification and Quality Control via Triacylglycerol (TAG) Profiles from Dry Matrix-Assisted Laser Desorption/Ionization Time-of-Flight (MALDI-TOF) Mass Spectrometry Fingerprinting. Journal of Agricultural and Food Chemistry, 57(10), 4030-4034.

Setzer, W. N.; Noletto, J. A.; \& Lawton, R. O. (2006). Chemical composition of the floral essential oil of Randia matudae from Monteverde, Costa Rica. Journal Flavor Frangr. 21, 244-246.

Silva Junior, J. F. (2004). A cultura da mangaba. Jaboticabal: Revista Brasileira de Fruticultura, 26 